\title{
Interpretation of self-potential anomaly over 2-D inclined thick sheet structures and analysis of uncertainty using very fast simulated annealing global optimization
}

\author{
Arkoprovo Biswas $^{1,2}$ (D) Shashi Prakash Sharma ${ }^{2}$
}

Received: 2 November 2015/Accepted: 18 June 2016/Published online: 2 July 2016

(C) Akadémiai Kiadó 2016

\begin{abstract}
Global optimization for the interpretation of Self-Potential (SP) anomaly using a very fast simulated-annealing (VFSA) inversion code is developed in the present study. The SP anomaly is measured over a 2-D inclined thick sheet-type electrostatically polarized structure. Different model parameters for thick sheet-type structures are optimized. VFSA optimization yields a large number of well-fitting solutions in a huge model space. Uncertainty in the interpretation is analyzed and the study shows that it remains in few model parameters. The efficacy of the developed approach has been demonstrated using noise free and noisy synthetic examples. Field data from three different regions is interpreted for multiple thick sheet-type structures related to mineral exploration. The computation time for finding this convenient solution is very short (100 s for single structure) and the proposed method is found to be useful for interpretation of thick sheettype structure and for multiple bodies as well. The interpretation procedure can be used for assessing mineral deposits as well as the SP of the earth's crust.
\end{abstract}

Keywords Self-Potential · Interpretation · VFSA · Ambiguity · Mineralization

\section{Introduction}

Mineralization potentials are the indication of interest in mineral exploration. These are associated predominantly with massive sulphide ore bodies and have been explained using an electro-chemical mechanism (Sato and Mooney 1960) or related to oxidation potential

Arkoprovo Biswas

arkoprovo@gmail.com

1 Geophysics Group, Wadia Institute of Himalayan Geology, 33, GMS Road, Dehradun 248001, Uttarakhand, India

2 Department of Geology and Geophysics, Indian Institute of Technology Kharagpur, Kharagpur, WB 721302, India 
(Corry 1985). The technique has a varied kind of applications comprising mining fields (Sato and Mooney 1960; Heinrichs 1966; Logn and Bolviken 1974; Corry 1985), sulfide and graphite exploration (Sundararajan et al. 1998; Mendonca 2008); groundwater exploration (Monteiro Santos et al. 2002); geothermal exploration (Zlotnicki and Nishida 2003; Jardani et al. 2008; Mauri et al. 2010, 2012); mineral prospecting (El-Kaliouby and Al-Garni 2009; Mehanee et al. 2011; Peksen et al. 2011; Roudsari and Beitollahi 2013); environmental and engineering investigations (Nyquist and Corry 2002; Naudet et al. 2004 and Canton et al. 2010)

Interpretation of Self-potential data using different techniques has been proposed for many years. Interpretation of SP data can be performed using simple geometric model, forward modeling and inversion. Logical expression for simple geometrical shapes are given by El-Araby (2004) and El-Kaliouby and Al-Garni (2009). Many methods have been developed to interpret the SP anomaly assuming the body of simple geometry such as sphere, cylinder, thin and thick sheet (Abdelrahman et al. 2006a, b, 2008, 2009; Essa 2011). Lately, El-Kaliouby and Al-Garni 2009 developed the modular neural networks (MNN) method and Monteiro Santos (2010) used the particle swarm optimization method (PSO) method for the interpretation of SP anomaly. Fedi and Abbas (2013) also proposed a new method for the interpretation of SP anomaly using depth from extreme point (DEXP) method. Dmitriev (2012) also proposes a new software algorithm for interpretation of SP anomaly for different thick sheet type and prismatic bodies but the uncertainty in interpreting the different model parameters was not discussed. It is well known that the problem of ambiguity, non-uniqueness and resolution are characteristic in various interpretation approaches. Interpretation of SP anomaly also suffers from this drawback. Sharma and Biswas (2013), Biswas 2013 and Biswas and Sharma (2015) presented the ambiguity in the interpretation of SP data using sheet-type model and proposes the ambiguity associated with idealized bodies. In the present study, ambiguity associated with the interpretation of SP data over thick sheet-type structures is analyzed using VFSA global optimization method which has not yet attempted in any literature.

VFSA optimization scan a vast model space without compromising the resolution and it had been extensively used in many geophysical applications (Sen and Stoffa 2013; Sharma and Kaikkonen 1999; Sharma 2012; Sharma and Biswas 2013; Biswas and Sharma 2015; Biswas 2015, 2016). Therefore, model parameters of thick sheet-type structures are optimized in a vast model space and ambiguities are analyzed. The present work revolves around the development of efficient and accurate approach for interpretation of thick sheet

Fig. 1 Inclined 2-D thick sheet geometry (Dmitriev, 2012)

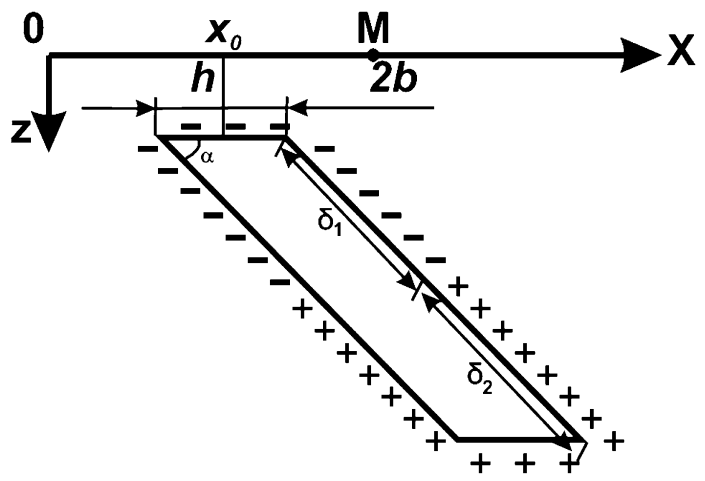


type structure, analysis of ambiguity in the interpretation and possible approach to avoid ambiguity for reliable result. The method is tested in synthetic and noise free data and for multiple sheet type structures for a field data.

\section{Forward formulation}

The SP anomaly $U_{M}$ at the point M on the surface due to a thick dipping layer (Fig. 1) is given by the equation (Dmitriev 2012).

$$
\begin{aligned}
U_{M} & =\frac{U_{0}}{\pi}\left[\left(2 \arctan \frac{-x+x_{0}-b+\delta_{1} \cos \alpha}{h+\delta_{1} \sin \alpha}+\arctan \frac{-x+x_{0}-b+\left(\delta_{1}+\delta_{2}\right) \cos \alpha}{h+\left(\delta_{1}+\delta_{2}\right) \sin \alpha}+\arctan \frac{x_{0}-x-b}{h}\right)\right. \\
& +\left(\arctan \frac{x_{0}-x+b}{h}-\arctan \frac{x_{0}-x-b}{h}\right) \\
& \left.\left(-2 \arctan \frac{x-x_{0}-b-\delta_{1} \cos \alpha}{h+\delta_{1} \sin \alpha}+\arctan \frac{x-x_{0}-b-\left(\delta_{1}+\delta_{2}\right) \cos \alpha}{h+\left(\delta_{1}+\delta_{2}\right) \sin \alpha}+\arctan \frac{x-x_{0}-b}{h}\right)\right]
\end{aligned}
$$

In the above equation $U_{0}$ is the self-potential of the layer/polarization parameter (negative in case of negative anomalies); $h$ is the depth to the layer top; $b$ is the half-width (horizontal size) of the upper edge; $x_{0}$ corresponds to center of the upper edge along the $\mathrm{x}$-axis; $x$ corresponds to the point $\mathrm{M}$ along the $\mathrm{x}$-axis for which the potential is calculated; $\alpha$ is the dip angle; $\delta_{1}, \delta_{2}$ are the lengths of the negative and positive sides, respectively.

\section{Very fast simulated annealing (VFSA) global optimization}

\subsection{Theory}

Various global optimization methods are based on different principles such as Boltzmann's law of statistical mechanics, to reach the minimum energy state (simulated annealing), biological evolution (genetic algorithm and neural network), and natural behavior of an individual or a group to follow an optimum path (Particle Swarm Optimizations) (Sen and Stoffa 2013; Sharma and Kaikkonen 1998, 1999; El-Kaliouby and Al-Garni 2009; Monteiro Santos 2010; Sharma and Biswas 2011, 2013; Biswas and Sharma 2015; Singh and Biswas 2015). These methods overcome various limitations of linearized inversions (Sen and Stoffa 2013) such as stability and resolution, reduction in the non-uniqueness, etc. In the present study, a variant of simulated annealing (SA), i.e. VFSA, is used for the optimization of model parameters for various standard geometrical structures associated with self-potential anomalies. VFSA is more efficient than the conventional SA approach in terms of less CPU time, memory and resolution (Ingber and Rosen 1992). Simulated Annealing (SA) is a guided random-search technique which exploits an analogy between the way in which a metal cools and freezes into a minimum energy crystalline structure (the annealing process) and the search for a minimum in a more general mathematical system; it forms the basis of an optimization technique for combinatorial and other problems. However, this requires a large computing time. Fast annealing and very fast simulated annealing (VFSA) or even adaptive simulated annealing (ASA) are each in turn exponentially faster and overcome the problem of large computation time. The main difference between SA and VFSA is the faster cooling schedule in VFSA due to a sharper 
Cauchy probability distribution for the random selection of model parameters. Further, SA takes samples at the predefined interval that limits the model resolution, while VFSA can take any value in the model space and increase resolution. Further, VFSA does not remember all models in the optimization process, and hence needs very small memory. This approach has been used by various scientists for interpretation of different geophysical data (Zhao et al. 1996; Sharma and Kaikkonen 1998; 1999; Bhattacharya et al. 2003; Pei et al. 2009; Srivastava and Sen 2009; Li et al. 2011; Sharma and Biswas 2011). The VFSA optimization is also widely applied in non-geophysical problems and this shows efficacy of the VFSA optimization.

In global optimization techniques mentioned above, the misfit for a large number of models within a model space is computed. Next, probability of each model is computed and process tries to focus in the region of high probability. However, VFSA optimization does not computes misfit for a large number of models at a time. Instead it moves within the model space randomly and selects a new model, compute misfit and probability for that model. Then it selects or rejects the new model with respect to the previous model. The movement of model parameters in the vast model space follows Cauchy probability distribution, which has a sharper peak than Gaussian probability distribution. The shape distribution allows the temperature to be lowered at a much faster rate in VFSA than other conventional SA approaches and hence it reaches the final temperature rapidly.

Every geophysical inversion requires minimization of misfit between the measured and model data. Misfit or objective function is very important in every optimization and is also problem dependent (type/nature and magnitude of the observed response). The varying nature of the geophysical data requires different types of objective functions. VFSA or any global optimization technique requires a search range $\left(P_{i}^{\min }\right.$ and $P_{i}^{\max }$, minimum and maximum value of $i^{\text {th }}$ parameter or model space) for each model parameter, and during the process each parameter is optimized within the search range to find the best model that fits the observed response well. In the present study we find following misfit error $(\varphi)$ between the observed and model response suitable of SP data interpretation.

$$
\varphi=\frac{1}{N} \sum_{i=1}^{N}\left(\frac{V_{i}^{0}-V_{i}^{c}}{\left|V_{i}^{0}\right|+\left(V_{\max }^{0}-V_{\min }^{0}\right) / 2}\right)^{2}
$$

where, $N$ is number of data point, $V_{i}^{0}$ and $V_{i}^{\mathrm{c}}$ is the $i^{\text {th }}$ observed and model responses, $V_{\text {max }}^{0}$ and $V_{\text {min }}^{0}$ are the maximum and minimum value of observed response.

The details of VFSA process can be well understood from Sen and Stoffa (2013). The details of VFSA method has also been discussed by Sharma (2012), Sharma and Biswas (2013), Biswas and Sharma (2014a, b), Biswas and Sharma (2015) for resistivity sounding and ambiguity in self-potential interpretation.

\subsection{Global model and uncertainty analysis}

Different geophysical problems have different kinds of ambiguities and the estimation of a global model requires different approaches. VFSA optimization yields a number of goodfitting models and the global model can be predicted using different approaches. Various model parameters of good-fitting models may differ from each other and could lie in a wide range in the multidimensional model space. It is important to sample the region of model space where clustering occurs (where a large number of models are located). Different sampling techniques have been used to predict the global model and minimize uncertainty in the final model (Mosegaard and Tarantola 1995; Sen and Stoffa 2013). A detailed global 
model, uncertainty analysis can be found in various literature viz. Sharma (2012), Sharma and Biswas (2013), Biswas and Sharma (2014a, b), Biswas and Sharma (2015), Biswas (2015).

\subsection{Computation time}

In the present work, a simple desktop PC with Intel Core-2-Duo processor and MS FORTRAN Developer Studio are used to execute the developed program. Computations were performed based on idea given by Biswas and Sharma 2015; Biswas and Sharma 2014 a, b. The actual (not CPU) time for the whole computation process for a single structure is nearly $100 \mathrm{~s}$. However, for multiple structures, the computation time increases to about $40 \%$.

\section{Results}

\subsection{Synthetic example}

Initially, noise-free and noisy (20\% random noise) synthetic data sets were inverted to retrieve the actual model parametersusing VFSA global optimization. The synthetic data is generated using Eq. 1 for a number of models. These data sets were interpreted using VFSA global optimization to retrieve the actual model parameters. It is significant to highlight that once an adequately effective kind of noise is overlaid on synthetic data obtained for a model, we no longer know the actual model parameters. Therefore, first, noise-free data are interpreted to verify the efficacy of the approach in recovering the actual model parameters, and following this, noisy and field data are interpreted.

\subsubsection{Model 1}

Initially, the forward response is generated for Model 1 (Table 1) using Eq. 1. This data is inverted to retrieve the actual model parameters. After selecting a suitable search range for each model parameter, a single VFSA run is performed to check the appropriate convergence of each model parameter and misfit. We found that on a large search range, the model parameters $\left(h, \delta_{1}, \delta_{2}, U_{0}\right)$ cannot be determined precisely. Subsequently, we have reduced the search range on the basis of initial results and VFSA optimization is performed again. Convergence pattern of each model parameter and reduction of misfit is studied (Fig. 2).

Table 1 Actual model, search range and mean model for Model 1

\begin{tabular}{llcl}
\hline Parameters & Actual model & Search range & Mean model \\
\hline$x_{0}$ & 500 & $400-600$ & $500.0 \pm 0.4$ \\
$h$ & 50 & $20-80$ & $51.7 \pm 9.2$ \\
$b$ & 50 & $20-80$ & $49.5 \pm 0.7$ \\
$\delta_{1}$ & 100 & $50-150$ & $98.5 \pm 8.9$ \\
$\delta_{2}$ & 400 & $300-500$ & $398.9 \pm 16.6$ \\
$\alpha$ & 90 & $0-180$ & $90.0 \pm 0.3$ \\
$U_{0}$ & 750 & $700-800$ & $758.6 \pm 13.0$ \\
Misfit & & & $3.8 \times 10^{-8}$ \\
\hline
\end{tabular}


Fig. 2 Convergence pattern for all the parameters and misfit for Model 1
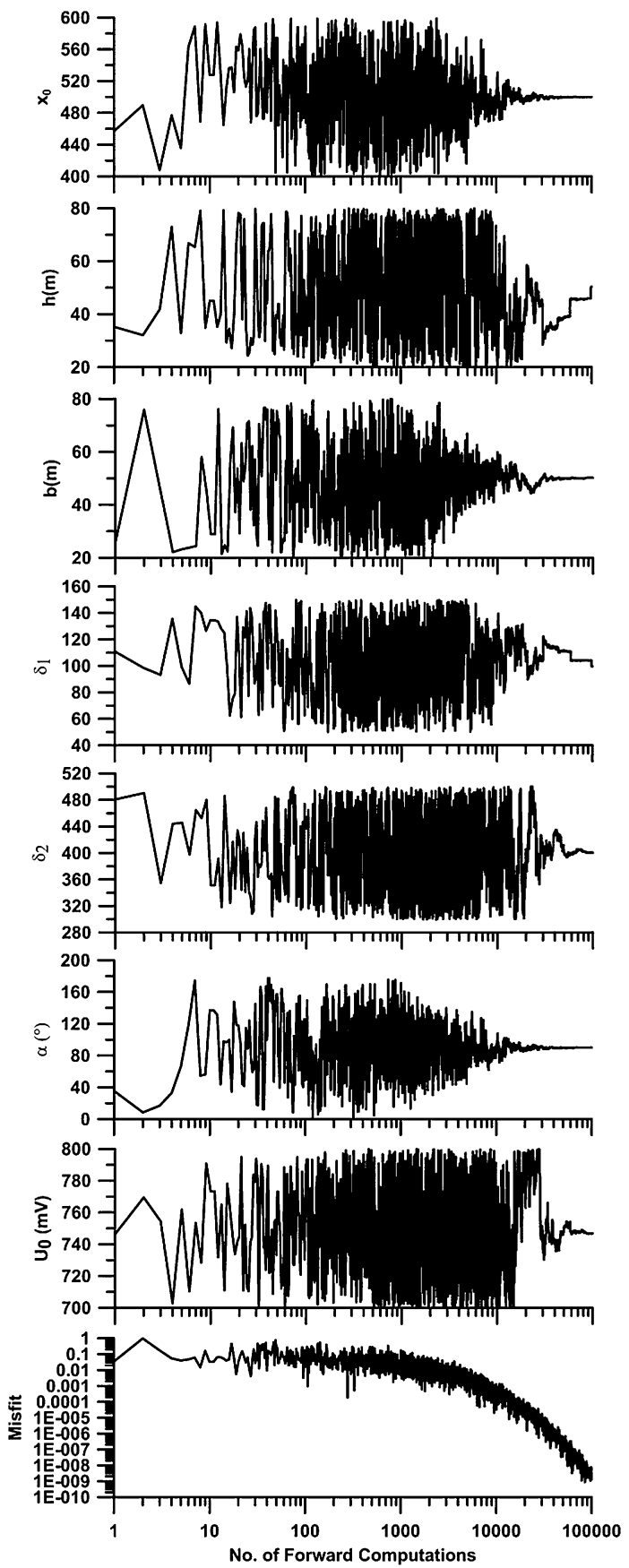

We can see that misfit fell to a small value systematically and that all model parameters converged to the actual values shown in Table 1 . It must be noted that misfit should show oscillations (decrease and increase) and finally steadily reach a very low value (Fig. 2). 
Figure 2 shows that various model parameters getting stabilized after 10,000 forward computations. However, iterations continue as the misfit error keeps on deceasing. Since these data are synthetic, the VFSA algorithm tried to reach the actual solution very accurately. Since various parameters keep getting refined up to several decimal places, the misfit decreases. However, in case of field data, it is always affected by noise and the condition could be different from that for synthetic data. Therefore, VFSA might need a larger number of repetitions.

Subsequently, ten VFSA runs are performed and 10 solutions are derived. Since $10^{6}$ models are evaluated (2000 iterations $\times 50$ number of moves at each iteration $\times 10$ VFSA runs) whose misfit vary from, $1-10^{-9}$ (noise free synthetic data), therefore, models having misfit greater than $10^{-5}$ are discarded. Figure 3 depicts the histogram of all accepted models whose misfit is lower than $10^{-4}$. Figures 3 depict that model parameter $x_{0}, b$ and $\alpha$ have a well-defined peak and are close to their actual value. However, other model parameters $h, \delta_{1}, \delta_{2}$ also shows a well-defined peak which is not close to the actual value and has a wide range of solutions. Parameter $U_{0}$ also shows a wide range of solutions from the histogram studies.

Next, Gaussian PDF is computed from models having misfit lower than $10^{-4}$. Finally only such models are selected to compute the statistical mean model and associated uncertainty whose all model parameters have PDF more than $60.65 \%$ (one standard deviation). We can see that the all model parameters except $U_{0}$ follows a Gaussian distribution however, $h, \delta_{1}, \delta_{2}$ shows that the PDF is not close to the actual initial value of that model parameter. Interestingly, $U_{0}$ show that the PDF is nearly close to the actual value. Hence, interpreted model parameters $h, \delta_{1}, \delta_{2}$ are ambiguous.

Figure 4 shows comparison between the observed and mean model data. Figure 4 shows the comparison between actual and modeled subsurface structure. Table 1 depicts the interpreted mean model and associated uncertainty. From the histogram and PDF, it can be concluded that the model parameters $x_{0}, b, \alpha$ and $U_{0}$ can be uniquely determined after global optimization and there is no ambiguity in the resolution of these parameters. However, there is ambiguity in the other model parameters $h, \delta_{1}, \delta_{2}$. The interpreted value of different parameter $\left(U_{0}, b, h, \alpha\right)$ increases while, both the poles of the thick type body decreases. This suggests that the depth from the top increases and the length of the body decreases. However, the amount of deviation from the initial model parameters is less than $10^{-8}$. It is interesting to note that for sheet type structures, depth from the top $(h)$ also pose ambiguity as well as the half width ( $a$ ) of the sheet (Sharma and Biswas 2013). There is a correlation between the parameter $h$ for both thin sheet-type and thick-sheet type structures which suggest that the depth of the body from the top of the surface remains uncertain.

Analysis of correlation matrix (Table 2) shows that when $h$ increases, then $U_{0}$ must increase, $\delta_{1}$ decreases and $\delta_{2}$ also slightly decreases. When $x_{0}$; increases $\alpha$ decrease. These are in accordance with the physics of the problem.
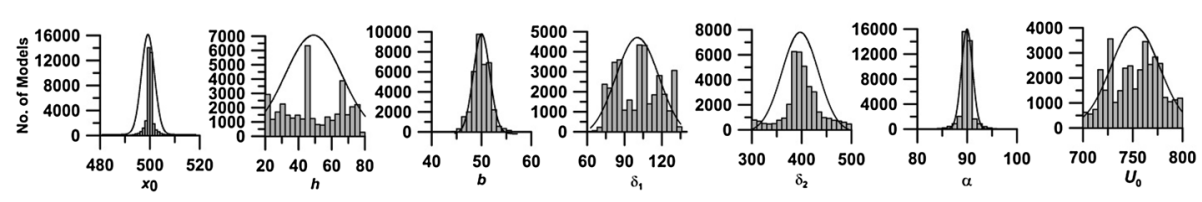

Fig. 3 Histogram of models having misfit $<10^{-4}$ and their PDFs, for Model 1 (noise-free synthetic data) 

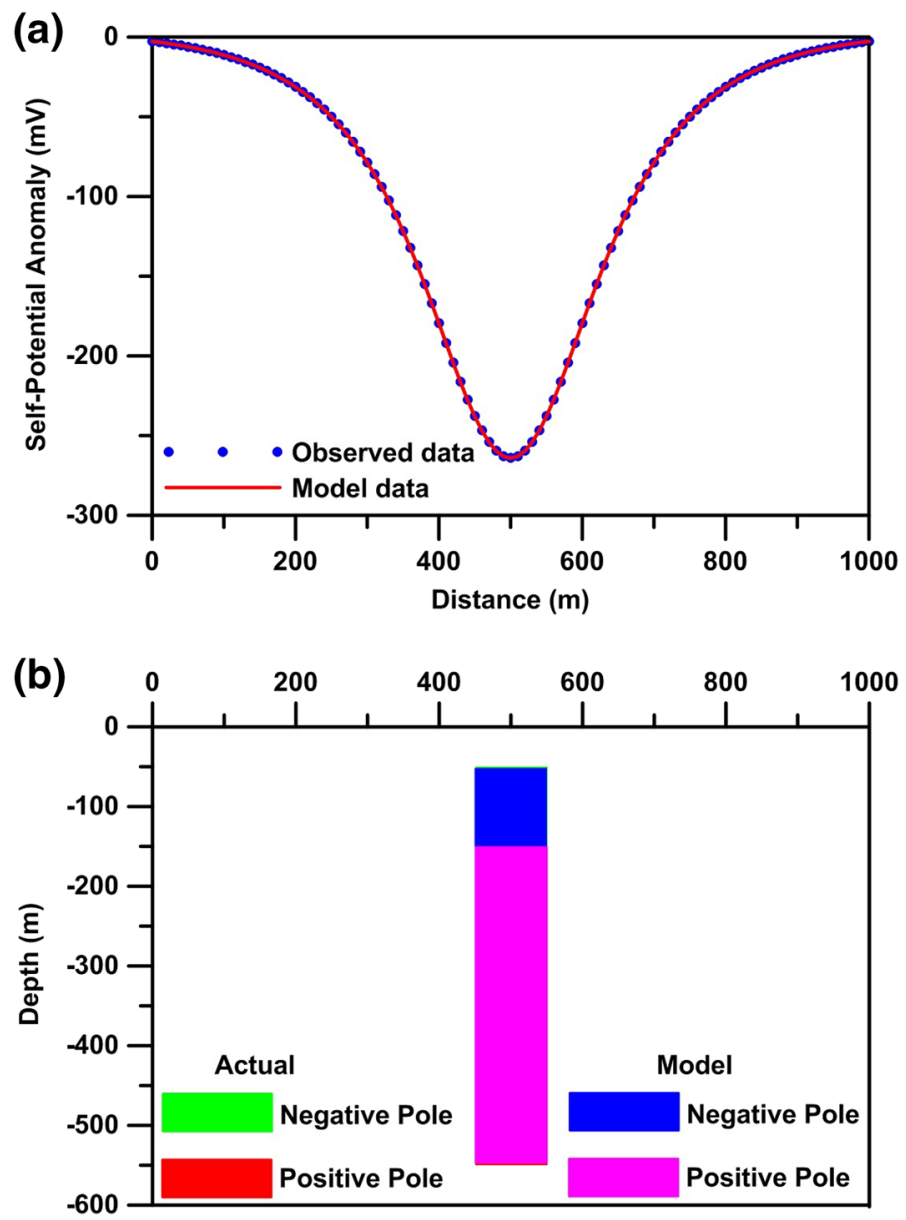

Fig. 4 Fitting between the observed and model response and corresponding subsurface structures for Model 1

Table 2 Correlation matrix for Model 1

\begin{tabular}{lllllllr}
\hline & \multicolumn{1}{c}{$x_{0}$} & \multicolumn{1}{l}{$h$} & \multicolumn{1}{l}{$b$} & \multicolumn{1}{c}{$\delta_{1}$} & \multicolumn{1}{c}{$\delta_{2}$} & \multicolumn{1}{l}{$\alpha$} & \multicolumn{1}{l}{$U_{0}$} \\
\hline$x_{0}$ & 1.000 & 0.042 & -0.092 & -0.038 & -0.067 & 0.827 & 0.121 \\
$h$ & & 1.000 & 0.002 & -0.996 & -0.382 & 0.083 & 0.781 \\
$b$ & & & 1.000 & -0.026 & 0.128 & -0.085 & 0.008 \\
$\delta_{1}$ & & & & 1.000 & 0.310 & -0.076 & -0.767 \\
$\delta_{2}$ & & & & & 1.000 & -0.100 & -0.459 \\
$\alpha$ & & & & & & 1.000 & 0.138 \\
$U_{0}$ & & & & & & & 1.000 \\
\hline
\end{tabular}


Table 3 Actual model, search range and mean model for Model 2

\begin{tabular}{llll}
\hline Parameters & Actual model & Search range & Mean model \\
\hline$x_{0}$ & 500 & $400-600$ & $530.2 \pm 11.9$ \\
$h$ & 80 & $20-100$ & $86.4 \pm 8.7$ \\
$b$ & 60 & $20-100$ & $81.7 \pm 7.3$ \\
$\delta_{1}$ & 120 & $50-150$ & $112.3 \pm 13.2$ \\
$\delta_{2}$ & 450 & $400-1000$ & $796.9 \pm 35.8$ \\
$\alpha$ & 120 & $90-180$ & $139.8 \pm 4.5$ \\
$U_{0}$ & 1000 & $600-1200$ & $616.9 \pm 64.0$ \\
Misfit & & & $1.3 \times 10^{-3}$ \\
\hline
\end{tabular}

\subsubsection{Model 2}

Forward response for Model 2 (Table 3) is computed using Eq. 1 and $20 \%$ uniformly random noise (i.e., multiplied by a random draw between 1 and 1.20) is added to the data. Then, VFSA optimization is performed in a way similar to the Model1. After studying the satisfactory convergence to obtain a single solution, ten VFSA runs are performed. Since the misfits for the evaluated $10^{6}$ models vary from $2-10^{-2}(20 \%$ uniformly random noisy synthetic data), models which have misfit less than $0.01 / 0.02$ are selected for statistical analysis based on the noise present in the data.

Figure 5 depict the histogram of all accepted models whose misfit is less than 0.02 and the Gaussian PDFs of models. The histogram reveals that Model 2 is similar in nature to Model 1. Additionally, the mean model is computed using models within the high PDF region. Again from the noisy data, it can be determined that the model parameters $x_{0}, b, \alpha$ and $U_{0}$ can be uniquely determined after global optimization and there is no ambiguity in the resolution of these parameters. However, the mean model for $h, \delta_{1}, \delta_{2}$ pose ambiguity as the histogram reveals that there is a wide solution for those model parameters and the mean model is not close to the actual value which is also in case for Model 1.

Figure 6 shows comparison between the observed and mean model data. Figure 6 shows the comparison between actual and modeled subsurface structure. Table 2 depicts the interpreted mean model and associated uncertainty. It is interesting to note that when the data is associated with noise, $h$ and $\delta_{2}$ increases whereas $\delta_{1}$ decreases. If $h$ increases, $\delta_{1}$ will decrease but $\delta_{2}$ should also slightly decrease. Since, the data is associated with noise; this kind of uncertainty may exist. However, the comparison between observed and mean model data show a good fit for noisy data. The subsurface model structure shows a deviation from the actual model which is due to the effect of noise in the data.

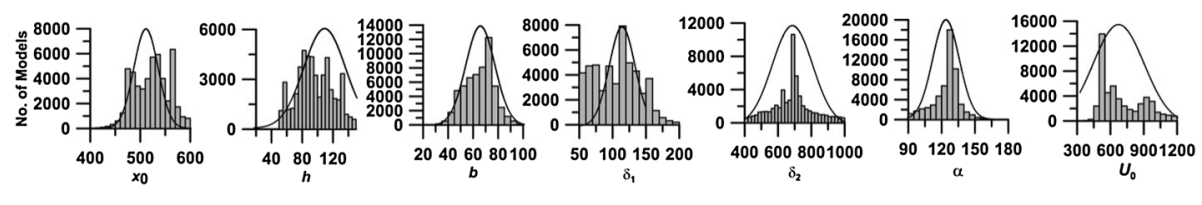

Fig. 5 Histogram of models having misfit $<0.02$ and their PDFs, for Model 2 (20\% uniformly random noisy synthetic data) 

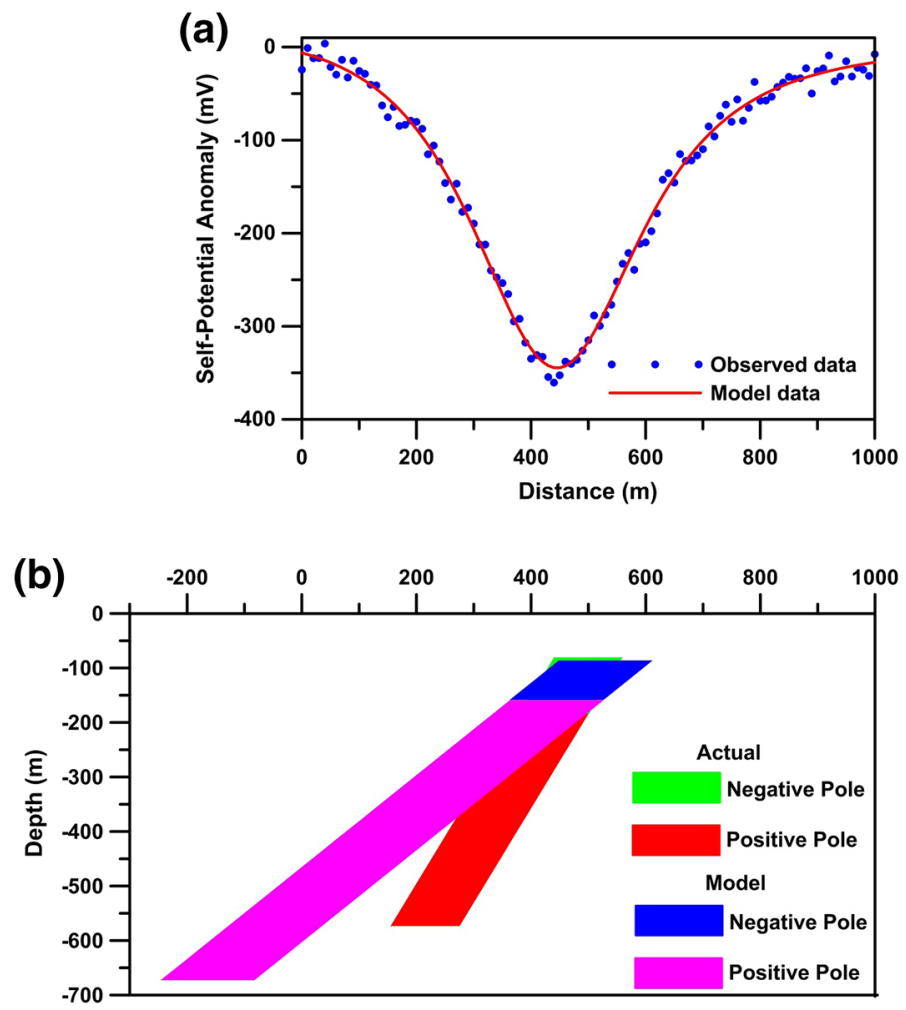

Fig. 6 Fitting between the observed and model response and corresponding subsurface structures for Model 2

\subsection{Field example}

\subsubsection{KTB anomaly, Bavaria, Germany}

Self-Potential anomaly (Fig. 7a) recorded from the vicinity of KTB -boreholes drilled during the German Continental Deep Drilling Program (Bigalke and Grabner 1997) in the northern part of Oberpfalz (NE Bavaria, Germany). The anomaly shows two negative peak anomaly zones. These two peak negative anomaly zones would be the favorable position to model the subsurface structure. Initially, a suitable search range for each model parameter is selected and a single VFSA run is performed to check the appropriate convergence of each model parameter and misfit. Next, Misfits for $10^{6}$ evaluated models vary from 2 down to $10^{-2}$, and we selected models with misfit smaller than 0.01 for statistical calculation.

Figure 7a shows the comparison between observed and model response. It can be seen that the model data fitted well for field data. Congruently, the subsurface structures have also been derived from the mean model parameters (Fig. 7b). The subsurface structures show the presence of two bodies after inversion of the field data using the thick sheet-type bodies. All the anomalies show a 2-D thick sheet-type structure (Fig. 8b). Both the structures show almost vertical in nature. The thickness of the first mineralized structure is $79.2 \mathrm{~m}$, length of the negative and positive pole of the body is 67 and $65.3 \mathrm{~m}$ respectively. The width of the second structure is $174.6 \mathrm{~m}$ and length of the negative and positive pole 

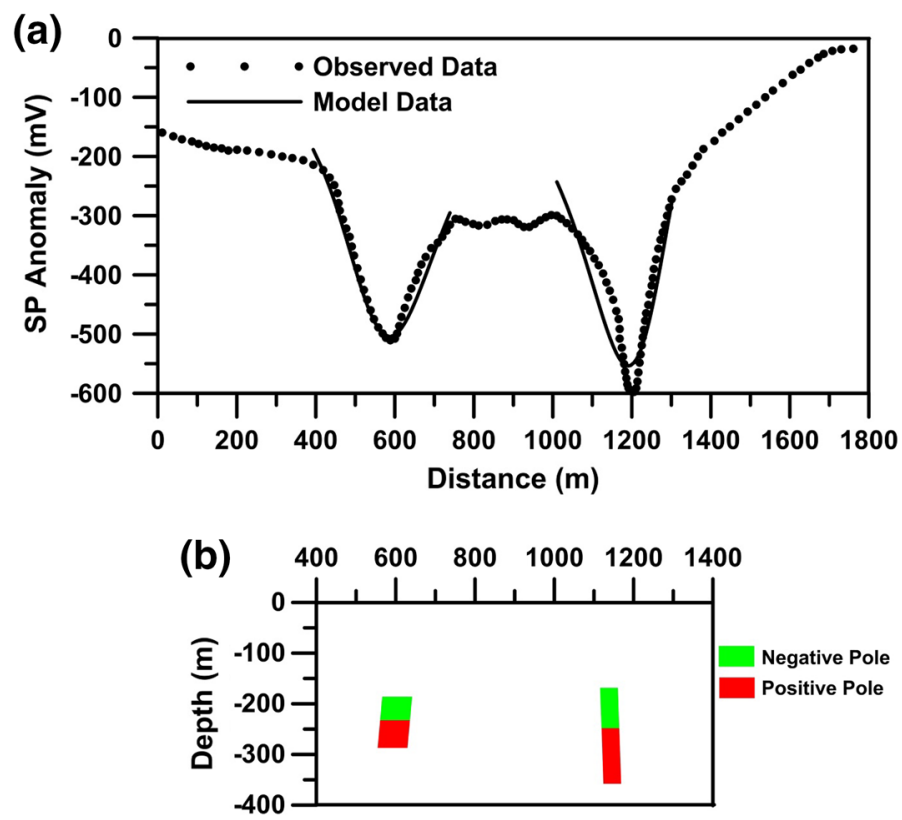

Fig. 7 Field example from KTB anomaly, Bavaria, Germany - Fitting between the observed and model response and corresponding subsurface structures

(a)

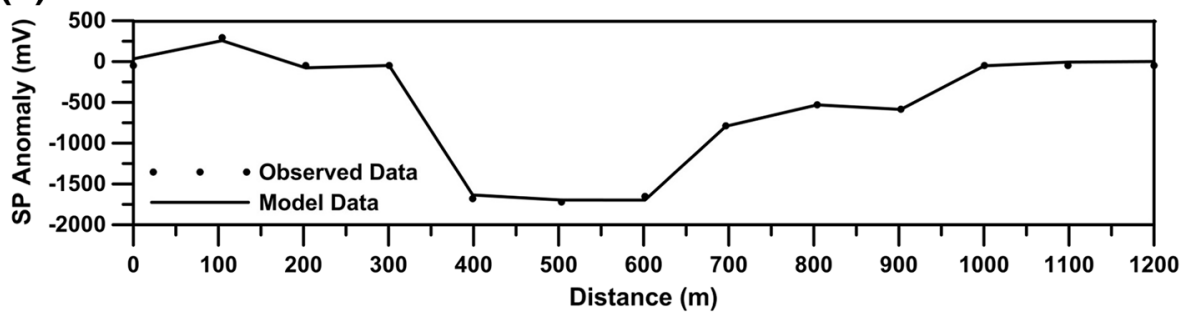

(b)

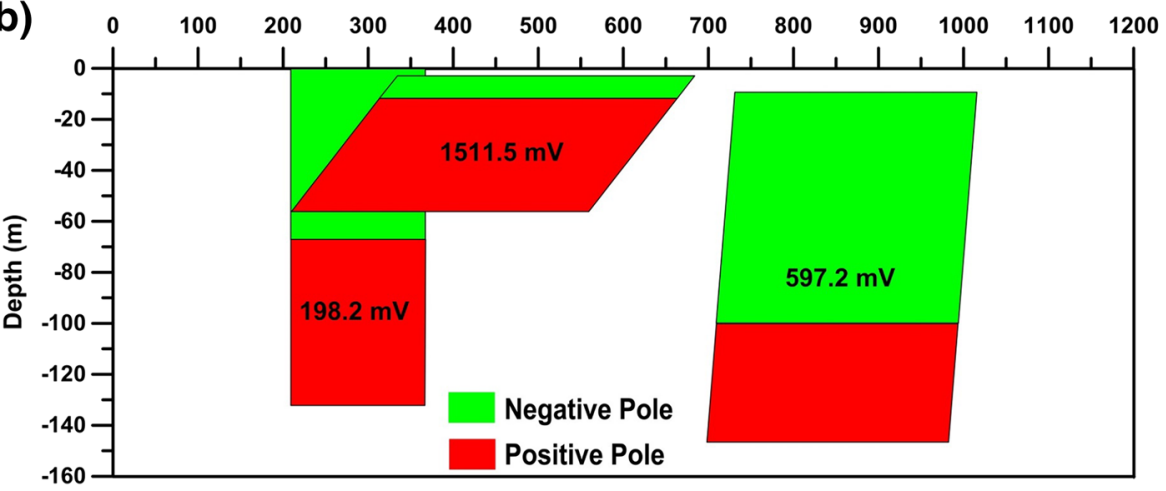

Fig. 8 Field example from Puca Rumi deposit, Peru - Fitting between the observed and model response and corresponding subsurface structures 
Table 4 Mean model for all two bodies at KTB anomaly, Bavaria, Germany

\begin{tabular}{llc}
\hline Parameters & Body 1 & Body 2 \\
\hline$x_{0}$ & $603.6 \pm 13.7$ & $1137.8 \pm 20.0$ \\
$h$ & $185.9 \pm 8.7$ & $168.2 \pm 8.8$ \\
$b$ & $37.4 \pm 6.9$ & $22.2 \pm 4.8$ \\
$\delta_{1}$ & $46.9 \pm 13.7$ & $79.9 \pm 19.9$ \\
$\delta_{2}$ & $57.6 \pm 7.5$ & $109.5 \pm 8.4$ \\
$\alpha$ & $174.4 \pm 12.2$ & $2.5 \pm 8.8$ \\
$U_{0}$ & $3632.7 \pm 616.5$ & $5063.7 \pm 1131.3$ \\
Misfit & $1.5 \times 10^{-7}$ & \\
\hline
\end{tabular}

of the body is 22.4 and $67.2 \mathrm{~m}$ respectively. The mean model parameters are shown in Table 4. It is highlighted that this field example was interpreted assuming thin sheet-type structure by Mehanee (2015). However, in the present work, it is interpreted as thick sheet type structure and is well correlated with the work of Dmitriev 2012.

\subsubsection{Puca rumi deposit, Peru}

Self-potential anomaly (Fig. 8a) was measured around Puca Rumi deposit, Peru. The anomaly is digitized at equal interval (after Mendonca 2008) for the present interpretation. The deposit is associated with silica ore bodies. From the anomaly (Fig. 8a), it can be seen that a multiple structure can be modeled from the observed data based on the peak negative anomaly (trough) zone. Hence, we tried to model this data considering three structures using the above mentioned approach.

Figure 8a shows the comparison between observed and model response. It can be seen that the model data fitted very well for field data. Correspondingly, the subsurface structures have been derived from the mean model parameters (Fig. 8b). The subsurface structures show the presence of three irregular bodies after inversion of the field data using the thick sheet-type bodies. All the anomalies show a 2-D thick sheet-type structure (Fig. 8b). The first structures show a vertical structure and the other two are dipping. The thickness of the first mineralized structure is $37.4 \mathrm{~m}$, length of the negative and positive pole of the body is 47 and $57.3 \mathrm{~m}$ respectively. The width of the second structure is $22 \mathrm{~m}$ and length of the negative and positive pole of the body is 80 and $109.5 \mathrm{~m}$ respectively. Interpreted polarization is shown in the figure itself (Fig. 8b) and the other model

Table 5 Mean model for all three bodies at Puca Rumi Deposit, Peru

\begin{tabular}{llrr}
\hline Parameters & \multicolumn{1}{l}{ Body 1 } & \multicolumn{1}{c}{ Body 2 } & \multicolumn{1}{c}{ Body 3 } \\
\hline$x_{0}$ & $288.2 \pm 0.0$ & $509.1 \pm 5.8$ & $873.5 \pm 8.9$ \\
$h$ & $0.02 \pm 0.0$ & $2.8 \pm 0.6$ & $9.5 \pm 1.2$ \\
$b$ & $79.2 \pm 0.6$ & $174.6 \pm 1.3$ & $142.0 \pm 0.5$ \\
$\delta_{1}$ & $67.0 \pm 2.5$ & $22.4 \pm 2.5$ & $93.2 \pm 3.5$ \\
$\delta_{2}$ & $65.3 \pm 0.8$ & $67.2 \pm 1.2$ & $47.7 \pm 0.6$ \\
$\alpha$ & $179.9 \pm 0.5$ & $113.1 \pm 0.5$ & $166.5 \pm 1.5$ \\
$U_{0}$ & $198.2 \pm 0.9$ & $1511.5 \pm 11.4$ & $597.2 \pm 8.4$ \\
& & & \\
Misfit & $5.0 \times 10^{-4}$ & & \\
\hline
\end{tabular}


(a)
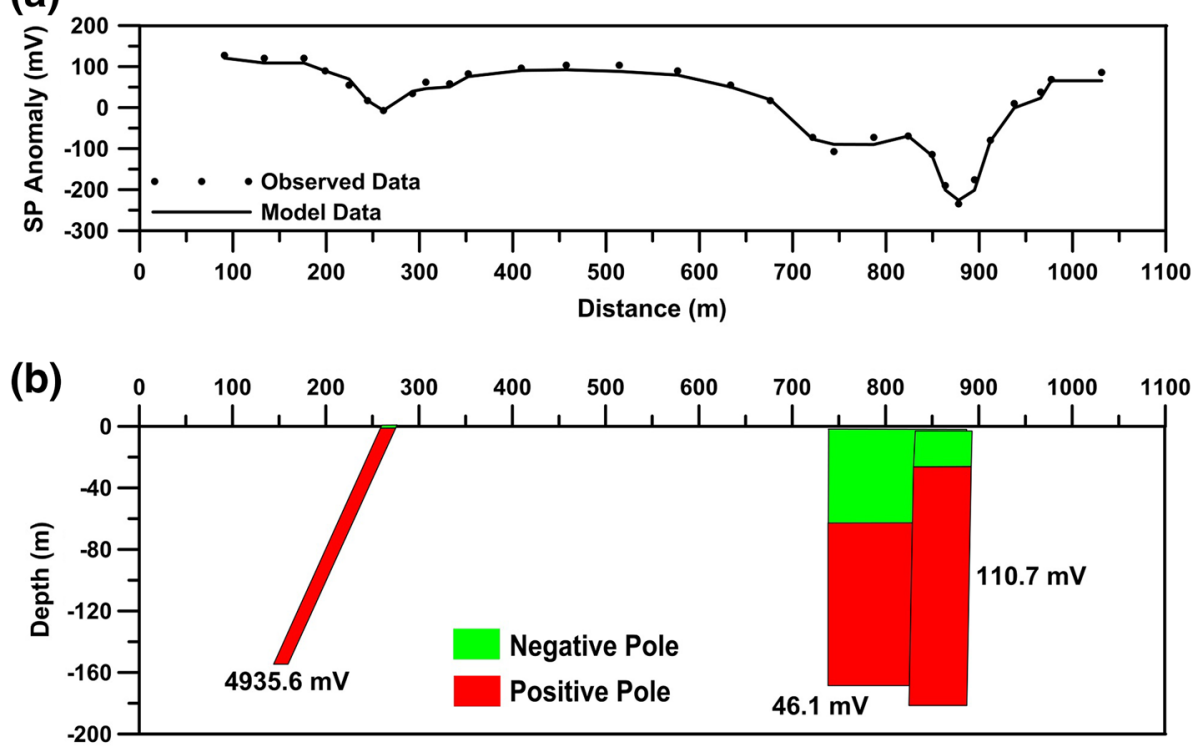

Fig. 9 Field example from Saurei deposits (Polar Urals) - fitting between the observed and model response and corresponding subsurface structures

parameters are shown in Table 5. However, the actual structure and presence of mineralization can be said from drilling information of that particular field area.

\subsubsection{Saurei deposit, Polar Urals}

Self-potential anomaly (Fig. 9a) was measured around a complex-ore field in the Polar Urals (after Khudyakov and Malivanchuk 1972). This ore body is related with lead deposits and presently known as Saurei Lead deposits. The anomaly is digitised at equal interval for the current interpretation. The anomaly (Fig. 9a), suggest that a multiple thick sheet-type structure can be modeled from the observed data based on the peak negative anomaly zone. Hence, we tried to model this data considering three structures and the methodology mentioned above.

Table 6 Mean model for all three bodies at Saurei Lead Deposit (Polar Urals)

\begin{tabular}{llrr}
\hline Parameters & Body 1 & \multicolumn{1}{c}{ Body 2 } & \multicolumn{1}{c}{ Body 3 } \\
\hline$x_{0}$ & $253.3 \pm 0.0$ & $812.7 \pm 12.7$ & $861.3 \pm 4.2$ \\
$h$ & $0.01 \pm 0.0$ & $1.8 \pm 0.9$ & $2.4 \pm 1.0$ \\
$b$ & $5.8 \pm 0.3$ & $74.0 \pm 0.6$ & $30.9 \pm 0.2$ \\
$\delta_{1}$ & $0.1 \pm 0.0$ & $61.0 \pm 12.4$ & $18.6 \pm 4.0$ \\
$\delta_{2}$ & $192.6 \pm 2.7$ & $105.7 \pm 6.3$ & $124.3 \pm 0.5$ \\
$\alpha$ & $143.4 \pm 0.7$ & $179.9 \pm 0.4$ & $2.3 \pm 0.4$ \\
$U_{0}$ & $4935.6 \pm 187.5$ & $46.1 \pm 0.7$ & $110.7 \pm 3.6$ \\
Misfit & $5.7 \times 10^{-3}$ & & \\
\hline
\end{tabular}


Figure 9a shows the comparison between observed and model response. It can be seen that the model data fitted very well for field data. Correspondingly, the subsurface structures have been derived from the mean model parameters (Fig. 9b). All the three anomalies show 2-D thick sheet-type structure. The first structures show an inclined structure and the other two are almost vertical. The thickness of the first mineralized structure is quite thin and is $5.8 \mathrm{~m}$, length of the negative and positive pole of the body is 0.1 and $192.6 \mathrm{~m}$ respectively. The width of the second structure is $74 \mathrm{~m}$ and length of the negative and positive pole of the body is 61 and $105.7 \mathrm{~m}$ respectively. The thickness of the third body is $142 \mathrm{~m}$. The length the negative and positive pole of the body is 93 and $47.7 \mathrm{~m}$ respectively. Interpreted polarization is shown in the figure itself (Fig. 9b) and the other model parameters are shown in Table 6.

It is highlighted that the field data is also interpreted using multiple thin sheet (after Biswas and Sharma 2014b; Sharma and Biswas 2013), sphere and cylindrical structures (after Biswas and Sharma 2015). However, the fittings are extremely poor and the model parameters are highly erroneous. Hence, the field data were modeled using 2-D thick sheettype structures.

It is also emphasized that actual structures may not have the standard shape (horizontal cylinder, vertical cylinder or thin and thick sheet-type) or structures in nature. Hence, modeling and inversion of actual field data with the above mentioned standard geometrical structures may not yield the actual subsurface structure. Slight deviation of the actual structure from the modeled structure (Sphere, cylinder, sheet, etc.) can be assumed to be superposition of diverse kind of noises on the responses generated by standard geometrical structures. However, we get a good estimate of the subsurface structure of a mineralized body and the location and depth of the body. It is also highlighted that the present method has been applied in interpretation of SP data from South Purulia Shear Zone (SPSZ) related to mineralization (Biswas et al. 2014; Biswas and Sharma 2016); however, the ambiguity associated with the interpretation was not discussed in that work. In the present work, ambiguity associated with the interpretation is also discussed taking synthetic and field examples.

\section{Conclusion}

VFSA global optimization is performed to interpret self-potential anomaly measured over a two dimensional thick sheet-type structure. Since, VFSA optimization yields a number of best-fitted models in a vast model space; the nature of ambiguity in the interpretation is also investigated simultaneously. The study demonstrates that graphical fits between the observed and model data are equally good as well as the subsurface models. However, detailed analysis of the histograms and estimated uncertainty shows that there are some uncertainties in model parameters. The study demonstrates that there is no ambiguity in the resolution of the model parameter $x_{0}, b, \alpha$. However, model parameters $h, \delta_{1}, \delta_{2}$ shows ambiguity in resolving the model parameter. This ambiguity is basically deviation of the mean model from the actual model parameter. Analysis of $U_{0}$ from the histogram suggests a wide range of solutions. This also poses ambiguity in resolving the actual model parameters. However, the mean model derived from probability density function suggests that it is very close to the actual model parameter. Synthetic noise free, noisy and field examples are presented in the study to demonstrate the efficacy of the developed approach. Field data from three different regions is interpreted using the developed approach. Since 
the computation time is very small, the developed method can be used competently to interpret SP data obtained from relevant field studies.

Acknowledgments This work is carried out at IIT Kharagpur as a part of AB's Ph.D work who is thankful to MHRD, Govt. of India and IIT Kharagpur for the funds in the form of Research Scholarship.

\section{Compliance with ethical standards}

Conflict of interest The authors declare there is no conflict of interest.

Informed consent was obtained from all individual participants included in the study The authors declare that this article does not contain any studies with human participants or animals performed by any of the authors.

Informed consent Informed consent was obtained from all individual participants included in the study.

\section{References}

Abdelrahman EM, Essa KS, Abo-Ezz ER, Soliman KS (2006a) Self-potential data interpretation using standard deviation of depths computed from moving-average residual anomalies. Geophys Prospect 54:409-423

Abdelrahman EM, Essa KS, Abo-Ezz ER, Soliman KS (2006b) A least-squares depth-horizontal position curves method to interpret residual SP anomaly profiles. J Geophys Eng 3:252-259

Abdelrahman EM, Essa KS, Abo-Ezz ER, Sultan M, Sauck WA, Gharieb AG (2008) New least-squares algorithm for model parameters estimation using self-potential anomalies. Comput Geosci 34:1569-1576

Abdelrahman EM, El-Araby TM, Essa KS (2009) Shape and depth determinations from second moving average residual self-potential anomalies. J Geophys Eng 6:43-52

Bhattacharya BB, Shalivahan, Sen MK (2003) Use of VFSA for resolution, sensitivity and uncertainty analysis in 1D-DC resistivity and IP inversion. Geophys Prospect 51:393-408

Bigalke J, Grabner EW (1997) The geobattery model: a contribution to large scale electrochemistry. Electrochim Acta 42(23-24):3443-3452

Biswas A (2013) Identification and resolution of ambiguities in interpretation of self-potential data: analysis and integrated study around South Purulia Shear Zone, India. Ph.D Thesis, Department of Geology and Geophysics, Indian Institute of Technology Kharagpur, 199 pp, doi:http://www.idr.iitkgp.ac.in/xmlui/ handle/123456789/3247

Biswas A (2015) Interpretation of residual gravity anomaly caused by a simple shaped body using very fast simulated annealing global optimization. Geosci Front 6(6):875-893

Biswas A (2016) Interpretation of gravity and magnetic anomaly over thin sheet-type structure using very fast simulated annealing global optimization technique. Model Earth Syst Environ 2(1):30

Biswas A, Mandal A, Sharma SP, Mohanty WK (2014) Delineation of subsurface structure using selfpotential, gravity and resistivity surveys from South Purulia shear zone, India: implication to uranium mineralization. Interpretation 2(2):T103-T110

Biswas A, Sharma SP (2014a) Optimization of Self-Potential interpretation of 2-D inclined sheet-type structures based on Very Fast Simulated Annealing and analysis of ambiguity. J Appl Geophys 105:235-247

Biswas A, Sharma SP (2014b) Resolution of multiple sheet-type structures in self-potential measurement. J Earth Syst Sci 123(4):809-825

Biswas A, Sharma SP (2015) Interpretation of self-potential anomaly over idealized body and analysis of ambiguity using very fast simulated annealing global optimization. Near Surf Geophys 13(2):179-195

Biswas A, Sharma SP (2016) Integrated geophysical studies to elicit the structure associated with Uranium mineralization around South Purulia Shear Zone, India: a Review. Ore Geol Rev 72:1307-1326

Canton M, Anschutz P, Naudet V, Molnar N, Mouret A, Franceschi M, Naessens F, Poirier D (2010) Impact of solid waste disposal on nutrient dynamics in a sandy catchment. J Contam Hydrol 116:1-15

Corry CE (1985) Spontaneous potential associated with porphyry sulphide mineralization. Geophysics 50:1020-1034

Dmitriev AN (2012) Forward and inverse self-potential modeling: a new approach. Russ Geol Geophys 53:611-622 
El-Araby HM (2004) A new method for complete quantitative interpretation of self-potential anomalies. J Appl Geophys 55:211-224

El-Kaliouby HM, Al-Garni MA (2009) Inversion of self-potential anomalies caused by 2D inclined sheets using neural networks. J Geophys Eng 6:29-34

Essa KS (2011) A new algorithm for gravity or self-potential data interpretation. J Geophys Eng 8:434-446

Fedi M, Abbas MA (2013) A fast interpretation of self-potential data using the depth from extreme points method. Geophysics 78:E107-E116

Heinrichs WE (1966) Geophysical investigation, Ore Knob Mine, Ashe County, North Carolina. Hanson DA, Heinrichs WE, Holmer RC, MacDougall RE, Rogers Summer JS, Ward SH (eds) Mining geophysics volume 1, case histories, SEG, Tusla, pp 179-184

Ingber L, Rosen B (1992) Genetic algorithms and very fast simulated re-annealing - A comparison. Math Comput Model 16:87-100

Jardani A, Revil A, Boleve A, Dupont JP (2008) Three-dimensional inversion of self-potential data used to constrain the pattern of groundwater flow in geothermal fields. J Geophys Res Solid Earth 113:B09204

Khudyakov AP, Malivanchuk VV (1972) The Saurei Pb deposit (Polar Urals). In: Geology and mineral deposits of the near-polar and Polar Urals [in Russian]. Tyumen, pp 211-224 (ZapSibNIGNI Transactions, Issue 52)

Li JH, Feng DS, Xiao JP, Peng LX (2011) Calculation of all-time apparent resistivity of large loop transient electromagnetic method with very fast simulated annealing. J Central South Univ Technol $18: 1235-1239$

Logn O, Bolviken B (1974) Self-potentials at the Joma pyrite deposit. Geoexploration 12:11-28

Mauri G, Williams-Jones G, Saracco G (2010) Depth determinations of shallow hydrothermal systems by self-potential and multi-scale wavelet tomography. J Volcanol Geoth Res 191(3-4):233-244

Mauri G, Williams-Jones G, Saracco G, Zurek JM (2012) A geochemical and geophysical investigation of the hydrothermal complex of masaya volcano, Nicaragua. J Volcanol Geotherm Res 227-228:15-31

Mehanee S (2015) Tracing of paleo-shear zones by self-potential data inversion: case studies from the KTB, Rittsteig, and Grossensees graphite-bearing fault planes, Earth. Planets Space 67:14

Mehanee S, Essa KS, Smith PD (2011) A rapid technique for estimating the depth and width of a twodimensional plate from self-potential data. J Geophys Eng 8:447-456

Mendonca CA (2008) Forward and inverse self-potential modeling in mineral exploration. Geophysics 73:F33-F43

Monteiro Santos FA (2010) Inversion of Self-potential of Idealized bodies' anomalies using particle swarm optimization. Comput Geosci 36:1185-1190

Monteiro Santos FA, Almeida EP, Castro R, Nolasco M, Mendes-Victor L (2002) A hydrogeological investigation using EM34 and SP surveys. Earth Planets Space 54:655-662

Naudet V, Revil A, Rizzo E, Bottero J-Y, Bégassat P (2004) Groundwater redox conditions and conductivity in a contaminant plume from geoelectrical investigations. Hydrol Earth Syst Sci 8(1):8-22

Nyquist JE, Corry CE (2002) Self-potential: the ugly duckling of environmental geophysics. Lead Edge 21(5):446-451

Pei DH, Quirein JA, Cornish BE, Quinn D, Warpinski NR (2009) Velocity calibration of microseismic monitoring: a very vast simulated annealing (VFSA) approach for joint-objective optimization. Geophysics 74:47-55

Peksen E, Yas T, Kayman AY, Ozkan C (2011) Application of particle swarm optimization on self-potential data. J Appl Geophys 75(2):305-318

Roudsari MS, Beitollahi A (2013) Forward modelling and inversion of self-potential anomalies caused by 2D inclined sheets. Explor Geophys 44(3):176-184

Sato M, Mooney HM (1960) The electrochemical mechanism of sulfide self-potentials. Geophysics 25:226-249

Sen MK, Stoffa PL (2013) Global optimization methods in geophysical inversion, 2nd edn. Cambridge Publisher, London

Sharma SP (2012) VFSARES- A very fast simulated annealing FORTRAN program for interpretation of 1-D DC resistivity sounding data from various electrode array. Comput Geosci 42:177-188

Sharma SP, Biswas A (2011) Global nonlinear optimization for the estimation of static shift and interpretation of 1-D Magnetotelluric sounding data. Ann Geophys 54(3):249-264

Sharma SP, Biswas A (2013) Interpretation of self-potential anomaly over a 2D inclined structure using very fast simulated-annealing global optimization-an insight about ambiguity. Geophysics 78:WB3WB15

Sharma SP, Kaikkonen P (1998) Two-dimensional nonlinear inversion of VLF-R data using simulated annealing. Geophys J Int 133:649-668 
Sharma SP, Kaikkonen P (1999) Appraisal of equivalence and suppression problems in 1-D EM and DC measurements using global optimization and joint inversion. Geophys Prospect 47:219-249

Singh A, Biswas A (2015) Application of global particle swarm optimization for inversion of residual gravity anomalies over geological bodies with idealized geometries. Nat Resour Res 25(3):297-314. doi:10.1007/s11053-015-9285-9

Srivastava RP, Sen MK (2009) Fractal-based stochastic inversion of post-stack seismic data using very fast simulated annealing. J Geophys Eng 6:412-425

Sundararajan N, Srinivasa Rao P, Sunitha V (1998) An analytical method to interpret self-potential anomalies caused by 2D inclined sheets. Geophysics 63:1551-1555

Zhao LS, Sen MK, Stoffa PL, Frohlich C (1996) Application of Very Fast Simulated Annealing to the Determination of the Crustal Structure beneath Tibet. Geophys Prospect 125:355-370

Zlotnicki J, Nishida Y (2003) Review on morphological insights of Self-Potential anomalies on volcanoes. Surv Geophys 24:291-338 